PROCEEDINGS OF THE

AMERICAN MATHEMATICAL SOCIETY

Volume 130, Number 9 , Pages 2701-2710

S 0002-9939(02)06386-4

Article electronically published on March 12, 2002

\title{
OPERATORS WHICH HAVE A CLOSED QUASI-NILPOTENT PART
}

PIETRO AIENA, MARIA LUISA COLASANTE, AND MANUEL GONZÁLEZ

(Communicated by Joseph A. Ball)

\begin{abstract}
We find several conditions for the quasi-nilpotent part of a bounded operator acting on a Banach space to be closed. Most of these conditions are established for semi-Fredholm operators or, more generally, for operators which admit a generalized Kato decomposition. For these operators the property of having a closed quasi-nilpotent part is related to the so-called single valued extension property.
\end{abstract}

\section{The QUASI-NILPOTENT PART OF AN OPERATOR AND THE SVEP}

The single valued extension property was first introduced by Dunford [5, [6] and has, successively, received a more systematic treatment in Dunford-Schwartz 7 . It also plays an important role in local spectral theory; see the monograph of Laursen and Neumann [14. The following local version of this property has been studied in recent papers by Aiena and Monsalve [1, 2] and previously by Finch [8].

Definition 1.1. Let $X$ be a complex Banach space and $T \in L(X)$. The operator $T$ is said to have the single valued extension property at $\lambda_{o} \in \mathbb{C}$ (abbreviated SVEP at $\lambda_{o}$ ), if for every open disc $\mathbf{D}_{\lambda_{o}}$ centered at $\lambda_{o}$ the only analytic function $f: \mathbf{D}_{\lambda_{o}} \rightarrow X$ which satisfies the equation $(\lambda I-T) f(\lambda)=0$ for all $\lambda \in \mathbf{D}_{\lambda_{o}}$ is the function $f \equiv 0$.

An operator $T \in L(X)$ is said to have the SVEP if $T$ has the SVEP at every point $\lambda \in \mathbb{C}$.

Let us consider the so-called local resolvent set $\rho_{T}(x)$ of $T$ at the point $x \in X$, defined as the union of all open subsets $\mathcal{U}$ of $\mathbf{C}$ such that there exists an analytic function $f: \mathcal{U} \rightarrow X$ which satisfies $(\lambda I-T) f(\lambda)=x$ for all $\lambda \in \mathcal{U}$. The local spectrum $\sigma_{T}(x)$ of $T$ at $x$ is the set defined by $\sigma_{T}(x):=\mathbb{C} \backslash \rho_{T}(x)$. Obviously, $\sigma_{T}(x) \subseteq \sigma(T)$, where $\sigma(T)$ denotes the spectrum of $T$.

For every subset $\Omega$ of $\mathbf{C}$, the analytic spectral subspace of $T$ associated with $\Omega$ is the set

$$
X_{T}(\Omega):=\left\{x \in X: \sigma_{T}(x) \subseteq \Omega\right\} .
$$

Received by the editors December 8, 2000 and, in revised form, April 20, 2001.

2000 Mathematics Subject Classification. Primary 47A10, 47A11; Secondary 47A53, 47A55.

Key words and phrases. Quasi-nilpotent part, single valued extension property, operators with a generalized Kato decomposition.

The research of the first two authors was supported by the International Cooperation Project between the University of Palermo (Italy) and Conicit-Venezuela.

The research of the third author was supported by DGICYT, Spain. 
It is easy to see from the definition that $X_{T}(\Omega)$ is a $T$-hyperinvariant linear subspace of $X[14]$.

The SVEP, as well as the SVEP at a point $\lambda_{o} \in \mathbf{C}$, may be characterized in a very simple way.

Theorem 1.2. Let $T \in L(X), X$ a Banach space. Then:

(i) $T$ has the SVEP at $\lambda_{o}$ if and only if $\operatorname{ker}\left(\lambda_{o} I-T\right) \cap X_{T}(\emptyset)=\{0\}$; see 11. Theorem 1.9].

(ii) $T$ has the $S V E P$ if and only if $X_{T}(\emptyset)=\{0\}$, and this is the case if and only if $X_{T}(\emptyset)$ is closed; see [14 Proposition 1.2.16].

Definition 1.3. Let $X$ be a Banach space and $T \in L(X)$. The analytical core of $T$ is the set $K(T)$ of all $x \in X$ such that there exists a sequence $\left(u_{n}\right)_{n \in \mathbb{N}} \subset X$ and $\delta>0$ for which $x=u_{o}, T u_{n+1}=u_{n}$ and $\left\|u_{n}\right\| \leq \delta^{n}\|x\|$, for every $n \in \mathbb{N}=\{0,1, \cdots\}$.

It is easy to check that $K(T)$ is a linear, generally not closed, subspace of $X$. Furthermore, $T(K(T))=K(T)$ and if $F$ is a closed subspace of $X$ for which $T(F)=F$, then $F \subseteq K(T)$; see [19, Proposition 2]. Note that if $T$ is quasinilpotent, then $K(T)=\{0\}$; see [16, Remarque 1.1].

Definition 1.4. Let $T \in L(X), X$ a Banach space. The quasi-nilpotent part of $T$ is the set

$$
H_{o}(T):=\left\{x \in X: \lim _{n \rightarrow \infty}\left\|T^{n} x\right\|^{\frac{1}{n}}=0\right\} .
$$

Also $H_{o}(T)$ is a linear subspace of $X$, generally not closed. Furthermore, $\operatorname{ker}\left(T^{m}\right) \subseteq H_{o}(T)$ for every $m \in \mathbb{N}$, and $T$ is quasi-nilpotent if and only if $H_{o}(T)=X$; see [20, Theorem 1.5].

The next result may be found in Vrbová 20, or Mbekhta [16; see also [14] Propositions 3.3.7 and 3.3.13].

Theorem 1.5. For a bounded operator $T \in L(X), X$ a Banach space, we have:

(i) $K\left(\lambda_{o} I-T\right)=X_{T}\left(\mathbb{C} \backslash\left\{\lambda_{o}\right\}\right)$.

(ii) $H_{o}\left(\lambda_{o} I-T\right) \subseteq X_{T}\left(\left\{\lambda_{o}\right\}\right)$ and the equality holds whenever $T$ has the SVEP.

In the sequel by $M^{\perp}$ we shall denote the annihilator of the subset $M \subseteq X$, and by ${ }^{\perp} N$ the pre-annihilator of the subset $N \subseteq X^{\star}$.

Theorem 1.6. For a bounded operator $T \in L(X), X$ a Banach space, the following implications hold:

(i) $H_{o}\left(\lambda_{o} I-T\right)$ closed $\Rightarrow H_{o}\left(\lambda_{o} I-T\right) \cap K\left(\lambda_{o} I-T\right)=\{0\} \Rightarrow T$ has the SVEP at $\lambda_{o}$.

(ii) $X=H\left(\lambda_{o} I-T\right)+K\left(\lambda_{o} I-T\right) \Rightarrow T^{\star}$ has the SVEP at $\lambda_{o}$.

Proof. Without loss of generality, we may consider $\lambda_{o}=0$.

(i) Assume that $H_{o}(T)$ is closed. Let $\tilde{T}$ denote the restriction of $T$ to the Banach space $H_{o}(T)$. Clearly, $H_{o}(T)=H_{o}(\tilde{T})$, thus $\tilde{T}$ is quasi-nilpotent. Therefore $K(\tilde{T})=\{0\}$. It is easy to see that $H_{o}(T) \cap K(T)=K(\tilde{T})$. This shows the first implication.

The second implication of (i) is an immediate consequence of Theorem 1.5. Indeed, we have

$$
\operatorname{ker}\left(\lambda_{o} I-T\right) \cap X_{T}(\emptyset) \subseteq H_{o}\left(\lambda_{o} I-T\right) \cap K\left(\lambda_{o} I-T\right),
$$


so, if the last intersection is $\{0\}$, then $T$ has the SVEP at $\lambda_{o}$, by Theorem 1.2

(ii) From [15, Proposition 1.8] we know that $H_{o}(T) \subseteq^{\perp} K\left(T^{\star}\right)$ and therefore $K\left(T^{\star}\right) \subseteq H_{o}(T)^{\perp}$. We also have $H_{o}\left(T^{\star}\right) \subseteq K(T)^{\perp}$. Indeed, let $\varphi \in H_{o}\left(T^{\star}\right)$ and consider an arbitrary element $x \in K(T)$. According to the definition of $K(T)$, there is a sequence $\left(u_{n}\right)_{n \in \mathbb{N}} \subset X$, and a $\delta>0$, such that $u_{o}=x, T u_{n+1}=u_{n}$ and $\left\|u_{n}\right\| \leq \delta^{n}\|x\|$ for every $n \in \mathbf{N}$. Clearly, $T^{n} u_{n}=x$ for every $n \in \mathbf{N}$. Consequently,

$$
|\varphi(x)|=\left|\varphi\left(T^{n} u_{n}\right)\right|=\left|\left(T^{\star n} \varphi\right)\left(u_{n}\right)\right| \leq\left\|u_{n}\right\|\left\|T^{\star n} \varphi\right\| \leq \delta^{n}\left\|T^{\star n} \varphi\right\|,
$$

and hence $|\varphi(x)|^{\frac{1}{n}} \leq \delta\left\|T^{\star n} \varphi\right\|^{\frac{1}{n}}$ for every $n \in \mathbf{N}$. The last term converges to 0 as $n \rightarrow \infty$, since $\varphi \in \bar{H}_{o}\left(T^{\star}\right)$, and from this it follows that $\varphi(x)=0$, i.e. $\varphi \in K(T)^{\perp}$. Finally, if $X=H_{o}(T)+K(T)$, then $\{0\}=H_{o}(T)^{\perp} \cap K(T)^{\perp} \supseteq H_{o}\left(T^{\star}\right) \cap K\left(T^{\star}\right)$. Thus, by part (i), $T^{\star}$ has the SVEP at 0 .

The two implications of part (i) of Theorem 1.6] were observed in [16, Proposition 1.10]. Later we shall prove that in the case of semi-Fredholm operators, or more generally in the case that $\lambda_{o} I-T$ admits a generalized Kato decomposition, the implications of Theorem 1.6 are actually equivalences.

Theorem 1.6 suggests in a very natural way the following definition:

Definition 1.7. A bounded operator $T \in L(X), X$ a Banach space, is said to have property $(Q)$ if $H_{o}(\lambda I-T)$ is closed for every $\lambda \in \mathbf{C}$.

Recall that a bounded operator $T \in L(X), X$ a Banach space, is said to have Dunford's property $(C)$, shortly property $(C)$, if the analytic subspace $X_{T}(\Omega)$ is closed for every closed subset $\Omega \subseteq \mathbb{C}$. From part (ii) of Theorem 1.2 it follows that if $T \in L(X)$ has property $(C)$, then $T$ has the SVEP.

An obvious consequence of part (ii) of Theorem 1.5 is that if $T$ has property $(C)$, then $H_{o}(\lambda I-T)=X_{T}(\{\lambda\})$ is closed for every $\lambda \in \mathbf{C}$, so that the following implications hold:

$$
T \text { has property }(C) \Rightarrow T \text { has property }(Q) \Rightarrow T \text { has the SVEP. }
$$

Note that neither of the implications in (1) may be reversed in general. A first counterexample, which shows that the first implication is not reversed in general, may be found among the convolution operators of group algebras.

Recall that a Banach algebra $A$ is said to be semi-simple if the radical rad $A=\{0\}[3 ; A$ is said to be semi-prime if there is no non-zero two-sided ideal $J$ for which $J^{2}=\{0\}$. Note that $A$ is semi-prime if and only if, for every $x \in A$, the identity $x A x=\{0\}$ implies that $x=0$, and that a commutative algebra is semi-prime if and only if it contains no non-zero nilpotent elements. Clearly, any semi-simple Banach algebra is semi-prime. A map $T: A \rightarrow A, A$ a Banach algebra, is said to be a multiplier if $(T x) y=x(T y)$ holds for all $x, y \in A$. Note that if $T$ is a multiplier of a semi-prime commutative Banach algebra $A$, then $(T x) y=x(T y)=$ $T(x y)$ for every $x, y \in A$; see the proof of [11, Theorem 1.1.1]. A very important example of a multiplier is given in the case that $A$ is the semi-simple Banach algebra $L_{1}(G)$, the group algebra of a locally compact abelian group $G$ with convolution as multiplication. Indeed, in this case to any complex Borel measure $\mu$ on $G$ there corresponds a multiplier $T_{\mu}$ defined by

$$
T_{\mu}(f):=\mu \star f \text { for all } f \in L_{1}(G),
$$


where

$$
(\mu \star f)(t):=\int_{G} f(t-s) d \mu(s) .
$$

The classical Helson-Wendel Theorem shows that each multiplier is a convolution operator and the multiplier algebra of $A:=L_{1}(G)$ may be identified with the measure algebra $M(G)$; see [11 Chapter 0].

Theorem 1.8. Let $T$ be a multiplier of a semi-simple Banach algebra A. Then

$$
H_{o}(T)=\operatorname{ker} T \text {. }
$$

In particular, $T$ has property $(Q)$.

Proof. We know that ker $T \subseteq H_{o}(T)$, so it remains to prove the inverse inclusion.

Suppose that $x \in H_{o}(T)$. By an easy inductive argument we have

$$
(T y)^{n}=\left(T^{n} y\right) y^{n-1} \text { for every } y \in A \text { and } n \in \mathbb{N} .
$$

From this it follows that

$$
\begin{aligned}
\left\|(a T x)^{n}\right\| & =\left\|(T a x)^{n}\right\|=\left\|T^{n}(a x)(a x)^{n-1}\right\| \\
& \leq\|a\|\left\|T^{n} x\right\|\left\|(a x)^{n-1}\right\|
\end{aligned}
$$

for every $a \in A$, so the spectral radius of the element $a T x$ satisfies

$$
r(a T x)=\lim _{n \rightarrow \infty}\left\|(a T x)^{n}\right\|^{\frac{1}{n}}=0
$$

for every $a \in A$. This implies that $T x \in \operatorname{rad} A ;$ see $[3, \S 25$, Proposition 1]. Since $A$ is semi-simple then $T x=0$ and therefore $H_{o}(T) \subseteq \operatorname{ker} T$.

The last assertion is clear, because $\lambda I-T$ is a multiplier of $A$ for every $\lambda \in \mathbf{C}$. (13]).

Clearly, if $T$ is a quasi-nilpotent multiplier, then $\operatorname{ker} T=H_{o}(T)=A$, so $T=0$

The following example shows that the assumption that $A$ is semi-simple in Theorem 1.8 cannot replaced by the weaker assumption that $A$ is semi-prime.

Example 1.9. Let $\omega:=\left(\omega_{n}\right)_{n \in \mathbb{N}}$ be a sequence with the property that $0<\omega_{m+n} \leq$ $\omega_{m} \omega_{n}$ for all $m, n \in \mathbb{N}$. Let $\ell^{1}(\omega)$ denote the space of all complex sequences $x:=$ $\left(x_{n}\right)_{n \in \mathbb{N}}$ for which $\|x\|_{\omega}:=\sum_{n=0}^{\infty} \omega_{n}\left|x_{n}\right|<\infty$. The space $\ell^{1}(\omega)$ equipped with convolution

$$
(x \star y)_{n}:=\sum_{j=0}^{n} x_{n-j} y_{j} \text { for all } n \in \mathbb{N}
$$

is a commutative unital Banach algebra. Let $A_{\omega}$ denote the maximal ideal of $\ell^{1}(\omega)$ given by

$$
A_{\omega}:=\left\{\left(x_{n}\right)_{n \in \mathbb{N}} \in \ell^{1}(\omega): x_{0}=0\right\} .
$$

The Banach algebra $A_{\omega}$ is an integral domain and hence semi-prime. Moreover, if the weight sequence $\omega$ satisfies the condition $\rho_{\omega}:=\lim _{n \rightarrow \infty} \omega_{n}^{\frac{1}{n}}=0$, then $A_{\omega}$ is a radical algebra ([14 Example 4.1.9]), i.e. coincides with its radical. Now, fix $0 \neq a \in A_{\omega}$ and let $T_{a}(x):=a \star x, x \in A_{\omega}$, denote the multiplication operator by the element $a$. From the estimate

$$
\left\|T^{n} x\right\|^{\frac{1}{n}}=\left\|a^{n} \star x\right\|^{\frac{1}{n}} \leq\left\|a^{n}\right\|^{\frac{1}{n}}\|x\|^{\frac{1}{n}}
$$


we see that $T_{a}$ is quasi-nilpotent, thus $H_{o}\left(T_{a}\right)=A_{\omega}$. On the other hand, $A_{\omega}$ is an integral domain so that $\operatorname{ker} T_{a}=\{0\}$.

Theorem 1.8 suggests the way of obtaining examples of operators which have property $(Q)$ but not property $(C)$. Indeed, there are convolution operators $T_{\mu}$, $\mu \in M(G)$, on the group algebra $L_{1}(G)$ which do not enjoy property $(C)$; see [14. Chapter 4].

The next example, which is obtained by a slight modification of Example 3.9 of [4], shows that also the second implication of (11) may be not reversed in general.

Example 1.10. Let $X:=\ell_{2} \oplus \ell_{2} \cdots$ and define

$$
T_{n} e_{i}:= \begin{cases}e_{i+1} & \text { if } i=1, \cdots, n \\ \frac{e_{i+1}}{i-n} & \text { if } i>n\end{cases}
$$

Clearly,

$$
\left\|T_{n}^{n+k}\right\|=\frac{1}{k !} \text { and }\left(\frac{1}{k !}\right)^{\frac{1}{n+k}} \rightarrow 0 \text { as } k \rightarrow \infty .
$$

From this it follows that $\sigma\left(T_{n}\right)=\{0\}$. Moreover, $T_{n}$ is injective and the point spectrum $\sigma_{p}\left(T_{n}\right)$ is empty. Now, let $T:=T_{1} \oplus \cdots \oplus T_{n} \oplus \cdots$. From $\left\|T_{n}\right\|=1$, for every $n \in \mathbf{N}$, we obtain $\|T\|=1$. From $\sigma_{p}\left(T_{n}\right)=\emptyset$ it also follows that $\sigma_{p}(T)=\emptyset$. Take $x=\left(x_{n}\right) \in X$ with $x_{n}:=\frac{e_{1}}{n}$. We have

$$
\|x\|=\left(\sum_{n=1}^{\infty} \frac{1}{n^{2}}\right)^{\frac{1}{2}}<\infty
$$

thus $x \in X$. Moreover,

$$
\left\|T^{n} x\right\|^{\frac{1}{n}} \geq\left\|T_{n}^{n} \frac{e_{1}}{n}\right\|^{\frac{1}{n}}=\left(\frac{1}{n}\right)^{\frac{1}{n}}
$$

and the last term does not converge to 0 . Clearly, $x \notin H_{o}(T)$. Finally, $\ell_{2} \oplus \ell_{2} \cdots \oplus$ $\ell_{2} \oplus\{0\} \cdots \subset H_{o}(T)$, where the non-zero terms are $n$. Since $H_{o}(T)$ contains all sequences with only finitely many non-zero terms, it follows that $H_{o}(T)$ is dense in $X$. Since $H_{o}(T) \neq X$ then $H_{o}(T)$ is not closed, thus $T$ has not property $(Q)$. Note that the operator $T$ has the SVEP, since $\sigma_{p}(T)$ is empty.

\section{The CASE OF SEMI-Fredholm OpERATORS}

For every linear operator $T$ on a vector space $X$, let us consider the increasing sequence of kernels ker $T^{n}$ and the decreasing sequence of ranges $T^{n}(X)$.

Definition 2.1. Let $T$ be a linear operator on a vector space $X$. The generalized kernel of $T$ is defined by

$$
\mathcal{N}(T):=\bigcup_{n \in \mathbb{N}} \operatorname{ker} T^{n}
$$

The hyperrange of $T$ is defined by

$$
T^{\infty}(X):=\bigcap_{n \in \mathbb{N}} T^{n}(X) .
$$

Clearly, $T^{\infty}(X)$ is a $T$-invariant subspace and it is easily seen that, if $X$ is a Banach space, $K(T) \subseteq T^{\infty}(X)$. Moreover, for every $n \in \mathbf{N}$ we have

$$
\operatorname{ker}\left(\lambda_{o} I-T\right)^{n} \subseteq \mathcal{N}\left(\lambda_{o} I-T\right) \subseteq H_{o}\left(\lambda_{o} I-T\right) \text {. }
$$


Recall that $T$ is said to have finite ascent if $\mathcal{N}(T)=\operatorname{ker} T^{k}$ for some positive integer $k$. Clearly, in such a case there is a smallest positive integer $p=p(T)$ such that $\operatorname{ker} T^{p}=\operatorname{ker} T^{p+1}$. The positive integer $p$ is called the ascent of $T$. Analogously, $T$ is said to have finite descent if $T^{\infty}(X)=T^{k}(X)$ for some $k$. The smallest integer $q=q(T)$ such that $T^{q+1}(X)=T^{q}(X)$ is called the descent of $T$. It is possible to prove that if $p(T)$ and $q(T)$ are both finite, then $p(T)=q(T)$; see 9 Proposition 38.3].

Theorem 2.2 ([1]). For a bounded operator $T$ on a Banach space $X$ the following implications hold:

i) $p\left(\lambda_{o} I-T\right)<\infty \Rightarrow T$ has SVEP at $\lambda_{o}$.

ii) $q\left(\lambda_{o} I-T\right)<\infty \Rightarrow T^{\star}$ has SVEP at $\lambda_{o}$.

Hence each one of the two conditions $p\left(\lambda_{o} I-T\right)<\infty$ or $H_{o}\left(\lambda_{o} I-T\right)$ closed implies the SVEP at $\lambda_{o}$. In general these two conditions are not related. The operator $T$ of Example 1.10 has ascent $p(T)=0$ and quasi-nilpotent part $H_{o}(T)$ not closed. In the following example we find an operator $T$ which has a closed quasi-nilpotent part but ascent $p(T)=\infty$.

Example 2.3. Let $T: \ell_{2} \rightarrow \ell_{2}$ be defined by

$$
T x:=\left(\frac{x_{2}}{2}, \cdots, \frac{x_{n}}{n}, \cdots\right) \text {, where } x=\left(x_{1}, \cdots, x_{n}, \cdots\right) .
$$

It is easily seen that $\left\|T^{k}\right\|=\frac{1}{(k+1) !}$ and from this it follows that $T$ is quasi-nilpotent and therefore $H_{o}(T)=\ell_{2}$. Obviouly, $p(T)=\infty$.

Definition 2.4. An operator $T \in L(X), X$ a Banach space, is said to be semiregular if $T(X)$ is closed and $\operatorname{ker} T \subseteq T^{\infty}(X)$.

An operator $T \in L(X)$ is said to admit a generalized Kato decomposition, abbreviated GKD, if there exists a pair of $T$-invariant closed subspaces $(M, N)$ such that $X=M \oplus N$, the restriction $T \mid M$ is semi-regular and $T \mid N$ is quasi-nilpotent.

Remark 2.5. In the sequel we list some examples of operators which admit a GKD.

(i) Every semi-regular operator has the GKD $M=X$ and $N=\{0\}$. Note that if $T$ is semi-regular, then $\overline{H_{o}(T)}=\overline{\mathcal{N}(T)}$; see [15, Proposition 2.10].

(ii) Every quasi-nilpotent operator has the GKD $M=\{0\}$ and $N=X$.

(iii) An important case is obtained if we assume in the definition above that $T \mid N$ is nilpotent. In this case $T$ is said to be of Kato type; see [15]. Obviously, any semi-regular operator is of Kato type. Note that if $T$ is of Kato type, then $T^{\infty}(X)=K(T)$ and $K(T)$ is closed; see [1, Lemma 2.4] or [2, Theorem 2.3 and Theorem 2.4].

(iv) Let

$$
\Phi_{+}(X):=\{T \in L(X): \operatorname{dim} \text { ker } T<\infty, T(X) \text { closed }\}
$$

denote the class of all upper semi-Fredholm operators, and let

$$
\Phi_{-}(X):=\{T \in L(X): \operatorname{codim} T(X)<\infty\}
$$

denote the class of all lower semi-Fredholm operators. The class of all semi-Fredholm operators is defined as $\Phi_{ \pm}(X):=\Phi_{+}(X) \cup \Phi_{-}(X)$ and the class of all Fredholm operators is defined as $\Phi(X):=\Phi_{+}(X) \cap \Phi_{-}(X)$. A well-known result of Kato [10] Theorem 4] establishes that every $T \in \Phi_{ \pm}(X)$ is of Kato type. More precisely, $T$ admits a $\operatorname{GKD}(M, N)$ with $T \mid N$ nilpotent and $\operatorname{dim} N<\infty$. 
Recall that for $T \in \Phi_{ \pm}(X)$ the index of $T$ is defined by ind $T:=\operatorname{dim} \operatorname{ker} T-$ codim $T(X)$. The index is an integer or $\pm \infty$.

Theorem 2.6. Let $T \in L(X), X$ a Banach space, and assume that $\lambda_{o} I-T$ has a $G K D(M, N)$. Then the following properties are equivalent:

(i) $T$ has the SVEP at $\lambda_{o}$.

(ii) $H_{o}\left(\lambda_{o} I-T\right) \cap K\left(\lambda_{o} I-T\right)=\{0\}$.

(iii) $H_{o}\left(\lambda_{o} I-T\right)$ is closed.

(iv) $H_{o}\left(\lambda_{o} I-T\right)=N$.

In particular, if $\lambda_{o} I-T$ is semi-regular the conditions (i)-(iv) are equivalent to the following one.

(v) $H_{o}\left(\lambda_{o} I-T\right)=\{0\}$.

Proof. Also here we only consider the case $\lambda_{o}=0$. Clearly, (iv) $\Rightarrow$ (iii) and from Theorem 1.6 we know that (iii) $\Rightarrow$ (ii) $\Rightarrow$ (i).

(i) $\Rightarrow$ (iv). First note that if $T$ admits a $\operatorname{GKD}(M, N)$, then $H_{o}(T)=H_{o}(T \mid M)+$ $H_{o}(T \mid N)$. The inclusion $H_{o}(T) \supseteq H_{o}(T \mid M)+H_{o}(T \mid N)$ is obvious. In order to show the opposite inclusion, consider an arbitrary element $x \in H_{o}(T)$ and let $x=u+v$, with $u \in M$ and $v \in N$. Since $T \mid N$ is quasi-nilpotent then $N=$ $H_{o}(T \mid N) \subseteq H_{o}(T)$. Consequently, $u=x-v \in H_{o}(T) \cap M=H_{o}(T \mid M)$ and therefore $H_{o}(T) \subseteq H_{o}(T \mid M)+H_{o}(T \mid N)$. Hence $H_{o}(T)=H_{o}(T \mid M)+H_{o}(T \mid N)=$ $H_{o}(T \mid M)+N$. Now, suppose that $T$ has the SVEP 0. Clearly, the SVEP at a point is inherited by the restrictions to closed invariant subspaces, so $T \mid M$ has the SVEP at 0 and from the semi-regularity of $T \mid M$ it follows that $T \mid M$ is injective; see [1, Theorem 2.14]. From this we obtain

$$
H_{o}(T \mid M)=\overline{\bigcup_{n=1}^{\infty} \operatorname{ker}(T \mid M)^{n}}=\{0\}
$$

and therefore $H_{o}(T)=N$.

The final assertion is clear.

Corollary 2.7. Let $T \in L(X), X$ a Banach space, and assume that $\lambda_{o} I-T$ is of Kato type. Then the conditions (i)-(iv) of Theorem 2.6 are equivalent to the following one:

$\left(\mathrm{v}^{\prime}\right) p\left(\lambda_{o} I-T\right)<\infty$.

In this case, if $p:=p\left(\lambda_{o} I-T\right)$, then

$$
H_{o}\left(\lambda_{o} I-T\right)=\mathcal{N}\left(\lambda_{o} I-T\right)=\operatorname{ker}\left(\lambda_{o} I-T\right)^{p} .
$$

Proof. Assume $\lambda_{o}=0$. We know that the inclusions $H_{o}(T) \supseteq \mathcal{N}(T) \supseteq$ ker $T^{n}$ hold for every $T \in L(X)$ and for every $n \in \mathbf{N}$. Let $(M, N)$ be a GKD for $T$ such that $(T \mid N)^{k}=0$ for some $k \in \mathbf{N}$. Then $H_{o}(T)=N \subseteq \operatorname{ker} T^{k}$ and hence $H_{o}(T)=\mathcal{N}(T)=\operatorname{ker} T^{k}$. From this it follows that $p:=p(T) \leq k$ and therefore ker $T^{k}=\operatorname{ker} T^{p}$.

The next result shows that, under the assumption that $\lambda_{o} I-T$ is semi-Fredholm, another equivalent condition can be added to those given in Corollary 2.7

Theorem 2.8. Suppose that $\lambda_{o} I-T \in L(X)$ is a semi-Fredholm operator. Then the following statements are equivalent:

(i) $H_{o}\left(\lambda_{o} I-T\right)$ is closed.

(ii) $H_{o}\left(\lambda_{o} I-T\right)$ is finite dimensional. 
Proof. We have only to prove the implication (i) $\Rightarrow$ (ii). This immediately follows from the mentioned Kato decomposition of a semi-Fredholm operator: if $(M, N)$ is a GKD for $\lambda_{o} I-T$ such that $\lambda_{o} I-T \mid N$ is nilpotent and $\operatorname{dim} N<\infty$, then $H_{o}\left(\lambda_{o} I-T\right)=N$, by Theorem [2.6.

The preceding result was obtained in [12, Theorem 2] under the assumption that $\lambda_{o} I-T$ is a Fredholm operator.

Theorem 2.9. Suppose that $\lambda_{o} I-T \in L(X)$ is of Kato type. Then the following statements are equivalent:

(i) $T^{\star}$ has the SVEP at $\lambda_{o}$.

(ii) $q:=q\left(\lambda_{o} I-T\right)<\infty$.

(iii) $X=H_{o}\left(\lambda_{o} I-T\right)+K\left(\lambda_{o} I-T\right)$.

Moreover, if any of the equivalent conditions (i)-(iii) holds, then

$$
\left(\lambda_{o} I-T\right)^{\infty}(X)=K\left(\lambda_{o} I-T\right)=\left(\lambda_{o} I-T\right)^{q}(X) .
$$

Proof. Assume that $\lambda_{o}=0$. The equivalence (i) $\Leftrightarrow$ (ii) has been proved in [2] Theorem 2.6]. The implication (iii) $\Rightarrow$ (i) has been proved in Theorem 1.6

(ii) $\Rightarrow$ (iii). Assume that $q:=q(T)<\infty$. Since $T$ is of Kato type then $K(T)=$ $T^{\infty}(X)=T^{q}(X)$. Moreover, $X=$ ker $T^{q}+T^{n}(X)$ for every $n \in \mathbf{N}$ (see [9] Proposition 38.2]), and therefore $X=H_{o}(T)+T^{\infty}(X)$.

Theorem 2.10. Suppose that $\lambda_{o} I-T \in L(X)$ is a semi-Fredholm operator. Then the following statements are equivalent:

(i) $T^{\star}$ has the SVEP at $\lambda_{o}$.

(ii) $K\left(\lambda_{o} I-T\right)$ is finite codimensional.

Proof. Also here we assume that $\lambda_{o}=0$.

(i) $\Rightarrow$ (ii). From Fredholm theory we know that $T^{\star}$ is also a semi-Fredholm operator and ind $T^{\star}=-$ ind $T$. Now, if $T^{\star}$ has the SVEP at 0 , then ind $T^{\star} \leq 0$ (see [1, Corollary 2.7]) and therefore ind $T \geq 0$. From this it follows that $T$ is a lower semi-Fredholm and consequently also $T^{q}$ is lower semi-Fredholm., i.e. $T^{q}(X)=T^{\infty}(X)=K(T)$ is finite codimensional.

(ii) $\Rightarrow$ (i). Since $K(T)=T^{\infty}(X)$, condition (ii) means that $T^{\infty}(X)$ is of finite codimension. But from this it is immediate that $q(T)<\infty$, so that $T^{\star}$ has the SVEP at 0 , by Theorem 2.9

It should be noted that if $T$ is semi-Fredholm, then $T^{\infty}(X)$ coincides with the socalled algebraic core of $T$, i.e. the greatest subspace $M$ of $X$ for which $T(M)=M$; see for instance [1, Theorem 2.3].

Corollary 2.11. Assume that $\lambda_{o} I-T \in L(X)$ is a semi-Fredholm operator. Then the following statements are equivalent:

(i) $T$ and $T^{\star}$ have the $S V E P$ at $\lambda_{o}$.

(ii) $X=H_{o}\left(\lambda_{o} I-T\right) \oplus K\left(\lambda_{o} I-T\right)$.

(iii) $H_{o}\left(\lambda_{o} I-T\right)$ is closed and $K\left(\lambda_{o} I-T\right)$ is finite-codimensional.

(iv) $\lambda_{o}$ is a pole of $(\lambda I-T)^{-1}$, or equivalently $p\left(\lambda_{o} I-T\right)=q\left(\lambda_{o} I-T\right)<\infty$.

(v) The spectrum does not cluster at $\lambda_{o}$.

In particular, if any of the equivalent conditions (i)-(v) holds and $p:=p\left(\lambda_{o} I-T\right)=$ $q\left(\lambda_{o} I-T\right)$, then

$$
H_{o}\left(\lambda_{o} I-T\right)=\mathcal{N}\left(\lambda_{o} I-T\right)=\operatorname{ker}\left(\lambda_{o} I-T\right)^{p}
$$


and

$$
K\left(\lambda_{o} I-T\right)=\left(\lambda_{o} I-T\right)^{\infty}(X)=\left(\lambda_{o} I-T\right)^{p}(X) .
$$

Proof. The equivalences of (i), (ii), (iii), and (iv) are obtained by combining all the results established in this section. The implication (iv) $\Rightarrow(\mathrm{v})$ is obvious. The implication (v) $\Rightarrow$ (i) is an immediate consequence of the fact that both $T$ and $T^{\star}$ have the SVEP at every point of the resolvent, as well as at every isolated point of the spectrum.

Note that rather similar results to those of Corollary 2.11 have been established by Mbekhta 15, Théorème 1.6] and Schmoeger [19, in the case that $\lambda_{o}$ is an isolated point of the spectrum.

Remark 2.12. Recall that for every $T \in L(X)$ the semi-Fredholm region is defined to be

$$
\Sigma(T):=\{\lambda \in \mathbb{C}: \lambda I-T \text { is semi-Fredholm }\} .
$$

It is well-known that $\Sigma(T)$ is an open set and hence it may be decomposed in connected disjoint open nonempty components. Suppose that $T$ has SVEP at some $\lambda_{o} \in \Omega, \Omega$ a component of $\Sigma(T)$. Then, by Theorem 2.6 and Corollary 2.7

$$
\begin{aligned}
\{0\} & =H_{o}\left(\lambda_{o} I-T\right) \cap K\left(\lambda_{o} I-T\right)=\mathcal{N}\left(\lambda_{o} I-T\right) \cap\left(\lambda_{o} I-T\right)^{\infty}(X) \\
& =\overline{\mathcal{N}\left(\lambda_{o} I-T\right)} \cap\left(\lambda_{o} I-T\right)^{\infty}(X),
\end{aligned}
$$

and from the constancy of the map $\lambda \in \Omega \rightarrow \overline{\mathcal{N}(\lambda I-T)} \cap(\lambda I-T)^{\infty}(X)$ (see 18 . Theorem 4.2]) we conclude that $\mathcal{N}(\lambda I-T) \cap(\lambda I-T)^{\infty}(X)=\{0\}$ for every point $\lambda \in \Omega$. From [2, Theorem 1.10] it follows that $T$ has the SVEP at every point $\lambda \in \Omega$. Moreover, the set

$$
\Gamma:=\{\lambda \in \Omega: \text { jump }(\lambda I-T) \neq 0\}
$$

is countable ([18]) and is equal to the set of all $\lambda \in \Omega$ such that $\lambda I-T$ is not semi-regular; see [21, Proposition 2.2]. From Theorem 2.6 then $H_{o}(\lambda I-T)=\{0\}$ for every $\lambda \in \Omega \backslash \Gamma$, while the remaining points $\lambda \in \Gamma$ are eigenvalues with ascent $p:=p(\lambda I-T)<\infty, H_{o}(\lambda I-T)=\operatorname{ker}(\lambda I-T)^{p}$ and $0<\operatorname{dim} H_{o}(\lambda I-T)<\infty$, by Corollary 2.7 and Theorem [2.8. In particular this situation occurs for every component of the semi-Fredholm region of an operator which has the SVEP.

\section{REFERENCES}

[1] P. Aiena, O. Monsalve Operators which do not have the single valued extension property. J. Math. Anal. Appl. 250 (2000), 435-448. MR 2001g:47005

[2] P. Aiena, O. Monsalve The single valued extension property and the generalized Kato decomposition property. Acta Sci. Math.(Szeged) 67 (2001), 461-477.

[3] F. F. Bonsall, J. Duncan Complete normed algebras. Springer-Verlag, Berlin, 1973. MR 54:11013

[4] I. Colojoară, C. Foiaş Theory of generalized spectral operators. Gordon and Breach, New York, 1968. MR 52:15085

[5] N. Dunford Spectral theory II. Resolution of the identity. Pacific J. Math. 2 (1952), 559-614. MR 14:479a

[6] N. Dunford Spectral operators. Pacific J. Math. 4 (1954), 321-354. MR 16:142d

[7] N. Dunford, J. T. Schwartz Linear operators, Part III. (1971), Wiley, New York. MR 54:1009

[8] J. K. Finch The single valued extension property on a Banach space. Pacific. J. Math. 58 (1975), 61-69. MR 51:11181

[9] H. Heuser Functional Analysis. (1982), Wiley, New York. MR 83m:46001 
[10] T. Kato Perturbation theory for nullity, deficiency and other quantities of linear operators. J. Anal. Math. 6 (1958), 261-322. MR 21:6541

[11] R. Larsen An introduction to the theory of multipliers. Springer-Verlag, New York, 1979. MR 55:8695

[12] K. B. Laursen, Essential spectra through local spectral theory. Proc. Amer. Math. Soc. 125 (1997), 1425-1434. MR 97g:46066

[13] K. B. Laursen, M. Mbekhta Closed range multipliers and generalized inverses. Studia Math. 107 (1993), 127-135. MR 94i:47052

[14] K. B. Laursen, M. M. Neumann An introduction to local spectral theory. Clarendon Press, Oxford, 2000. MR 2001k:47002

[15] M. Mbekhta Généralisation de la décomposition de Kato aux opérateurs paranormaux et spectraux. Glasgow Math. J. 29 (1987), 159-175. MR 88i:47010

[16] M. Mbekhta Sur la théorie spectrale locale et limite des nilpotents. Proc. Amer. Math. Soc. 110 (1990), 621-631. MR 91b:47004

[17] M. Mbekhta, A. Ouahab Opérateur s-régulier dans un espace de Banach et théorie spectrale. Acta Sci. Math. (Szeged) 59 (1994), 525-543. MR 96a:47018

[18] M. Ó Searcóid, T. T. West Continuity of the generalized kernel and range for semi-Fredholm operators. Math. Proc. Camb. Phil. Soc. 105 (1989), 513-522. MR 90d:47017

[19] C. Schmoeger On isolated points of the spectrum of a bounded operator. Proc. Amer. Math. Soc. 117 (1993), 715-719. MR 93d:47007

[20] P. Vrbová On local spectral properties of operators in Banach spaces. Czechoslovak Math. J. 23 (98) (1973), 483-92. MR 48:898

[21] T. T. West A Riesz-Schauder theorem for semi-Fredholm operators. Proc. Roy. Irish. Acad. 87 A (1987), 137-146. MR 89i:47020

Dipartimento di Matematica ed Applicazioni, Facoltà di Ingegneria, Università di Palermo, Viale delle Scienze, I-90128 Palermo, Italy

E-mail address: paiena@mbox.unipa.it

Departamento de Matemáticas, Facultad de Ciencias, Universidad de los Andes, Merida, Venezuela

E-mail address: marucola@ciens.ula.ve

Departamento de Matemáticas, Facultad de Ciencias, Universidad de Cantabria, SanTANDER, SPAIN

E-mail address: gonzalem@ccaix3.unican.es 